Algebraic 83 Geometric Topology

Volume 4 (2004) 1111-1123

Published: 25 November 2004

ATG

\title{
Alexander polynomial, finite type invariants and volume of hyperbolic knots
}

\author{
EFSTRATIA KALFAGIANNI
}

\begin{abstract}
We show that given $n>0$, there exists a hyperbolic knot $K$ with trivial Alexander polynomial, trivial finite type invariants of order $\leq n$, and such that the volume of the complement of $K$ is larger than $n$. This contrasts with the known statement that the volume of the complement of a hyperbolic alternating knot is bounded above by a linear function of the coefficients of the Alexander polynomial of the knot. As a corollary to our main result we obtain that, for every $m>0$, there exists a sequence of hyperbolic knots with trivial finite type invariants of order $\leq m$ but arbitrarily large volume. We discuss how our results fit within the framework of relations between the finite type invariants and the volume of hyperbolic knots, predicted by Kashaev's hyperbolic volume conjecture.
\end{abstract}

AMS Classification 57M25; 57M27, 57N16

Keywords Alexander polynomial, finite type invariants, hyperbolic knot, hyperbolic Dehn filling, volume.

\section{Introduction}

Let $c(K)$ denote the crossing number and let $\Delta_{K}(t):=\sum_{i=0}^{k} c_{i} t^{i}$ denote the Alexander polynomial of a knot $K$. If $K$ is hyperbolic, let $\operatorname{vol}\left(S^{3} \backslash K\right)$ denote the volume of its complement. The determinant of $K$ is the quantity $\operatorname{det}(K):=$ $\left|\Delta_{K}(-1)\right|$. Thus, in general, we have

$$
\operatorname{det}(K) \leq\left\|\Delta_{K}(t)\right\|:=\sum_{i=0}^{k}\left|c_{i}\right| .
$$

It is well know that the degree of the Alexander polynomial of an alternating knot equals twice the genus of the knot. On the other hand one can easily construct knots with arbitrarily large genus and trivial Alexander polynomial by taking connected sums of untwisted Whitehead doubles. In this note, we construct hyperbolic knots of arbitrarily large genus and volume that have trivial Alexander polynomial. To put our results into the appropriate context, let 
us first recall what is known about the relation between volume and Alexander polynomial of alternating knots. In general, it is known (see [6] and references therein) that there exists a universal constant $C>0$ such that if $K$ is a hyperbolic knot with crossing number $c(K)$, then

$$
\operatorname{vol}\left(S^{3} \backslash K\right) \leq C c(K) .
$$

If in addition $K$ is alternating then one also has ( [5])

$$
c(K) \leq \operatorname{det}(K) \text { and } \operatorname{det}(K)=\left\|\Delta_{K}(t)\right\| .
$$

By a result of Menasco [13, prime, alternating knots are either torus knots or hyperbolic. Combining this with (1)-(3), we derive that there is a universal constant $C>0$ such that we have

$$
\operatorname{vol}\left(S^{3} \backslash K\right) \leq C \operatorname{det}(K) \text { and } \operatorname{vol}\left(S^{3} \backslash K\right) \leq C\left\|\Delta_{K}(t)\right\|,
$$

for every prime, alternating non-torus knot $K$. (In fact, in [17, A. Stoimenow showed that the quantity $\operatorname{det}(K)$ in $(4)$ can be replaced by $\log (\operatorname{det}(K))$.) Thus, the Alexander polynomial of prime alternating knots, dominates two of the most important geometric knot invariants; namely the volume and the genus. In contrast to that, the main result in this paper implies the following.

Corollary 1.1 Given $n \in \mathbf{N}$, there exists a hyperbolic knot $K$ with trivial Alexander polynomial for which we have $\operatorname{vol}\left(S^{3} \backslash K\right)>n$ and $\operatorname{genus}(K)>\frac{n}{6}$.

The development of quantum topology led to many new knot invariants that generalized the Alexander polynomial. These are the Jones type polynomial invariants (quantum invariants) and their perturbative counterparts known as finite type invariants (or Vassiliev invariants). An open conjecture relating these invariants to the geometry of the knot complement is the hyperbolic volume conjecture of R. Kashaev [12. Kashaev's conjecture, as rephrased after the work of H. Murakami and J. Murakami [14, asserts that the volume of the complement of a hyperbolic knot $K$ is equal to a certain limit of values of the colored Jones polynomials of $K$. A convenient way to organize these polynomials is via the so-called colored Jones function. This is a 2-variable formal power series

$$
J_{K}(h, d)=\sum_{i \geq 0, j \leq i} a_{i j} h^{i} d^{j} \in \mathbf{Q}[h, d],
$$

such that $a_{i j}$ is a Vassiliev invariant of order $i$ for $K$. By the Melvin-MortonRozansky conjecture, the first proof of which was given in [3], the diagonal subseries $D_{K}(h):=\sum_{i} a_{i i} h^{i}$ is essentially equivalent to the Alexander polynomial of $K$. The volume conjecture implies that the colored Jones function 
of a hyperbolic knot $K$ determines the volume of the complement of $K$. In particular, it implies that if there exist two hyperbolic knots that have all of their finite type invariants the same then their complements should have equal volumes. In this setting, Corollary 1.1 says that the part $D_{K}(h)$ of $J_{K}(h, d)$ is far from controlling the volume of hyperbolic knots. The following theorem, which is the main result of the paper, provides a stronger assertion. It implies that there can be no universal value $n \in \mathbf{N}$ such that, for every hyperbolic knot $K$, the finite type invariants of orders $\leq n$ together with $D_{K}(h)$ determine the volume of the complement of $K$.

Theorem 1.2 For every $n \in \mathbf{N}$, there exists a hyperbolic knot $K$ such that:

(a) We have $\Delta_{K}(t)=1$.

(b) All the finite type invariants of orders $<2 n-1$ vanish for $K$.

(c) We have $\operatorname{vol}\left(S^{3} \backslash K\right)>n$.

(d) We have genus $(K)>\frac{n}{6}$.

Theorem 1.2 has the following corollary which implies that for every $m \in \mathbf{N}$, there are hyperbolic knots with arbitrarily large volume but trivial " $m$ - truncated" colored Jones function. The proof of the corollary is spelled out at the end of Section 3.

Corollary 1.3 For every $n, m \in \mathbf{N}$, there exists a hyperbolic knot $K$ with trivial finite type invariants of orders $\leq m$ and such that $\operatorname{vol}\left(S^{3} \backslash K\right)>n$.

The proof of Theorem 1.2 combines results of [2] and [10] with techniques from hyperbolic geometry [18]. Given $n \in \mathbf{N}$, 2] provides a method for constructing knots with trivial Alexander polynomial and trivial finite type invariants of orders $<2 n-1$. To obtain hyperbolicity and appropriate volume growth we combine a result from [10 with the work of Thurston and a result of Adams [1. The results of [2, [10] needed in this paper are summarized in Section 2 where we also prove some auxiliary lemmas. Section 3 is devoted to the proof of Theorem 1.2

Acknowledgment The author thanks Oliver Dasbach and Xiao-Song Lin for useful discussions and correspondence. She also thanks Alex Stoimenow for his interest in this work and Dan Silver for helpful comments and for informing her about his work [16. In addition, she acknowledges the support of the NSF through grants DMS-0104000 and DMS-0306995 and of the Institute for Advanced Study through a research grant. 


\section{Preliminaries}

In this section we recall results and terminology that will be used in the subsequent sections and we prove some auxiliary lemmas needed for the proof of the main results. A crossing disc for a knot $K$ is a disc $D$ that intersects $K$ only in its interior exactly twice with zero algebraic intersection number; the boundary $\partial D$ is called a crossing circle. Let $q \in \mathbf{Z}$. A knot $K^{\prime}$ obtained from $K$ by twisting $q$-times along $D$ is said to be obtained by a generalized crossing change of order $|q|$. Clearly, if $q=0$ we have $K=K^{\prime}$ and if $|q|=1$ then $K, K^{\prime}$ differ by an ordinary crossing change. Note that $K^{\prime}$ can also be viewed as the result from $K$ under $\frac{1}{q}$-surgery of $S^{3}$ along $\partial D$.

Definition 2.1 10] We will say that $K$ is $n$-adjacent to the unknot, for some $n \in \mathbf{N}$, if $K$ admits an embedding containing $n$ generalized crossings such that changing any $0<m \leq n$ of them yields an embedding of the unknot. A collection of crossing circles corresponding to these crossings is called an $n$-trivializer.

Let $V$ be a solid torus in $S^{3}$ and suppose that a knot $K$ is embedded in $V$ so that it is geometrically essential. We will use the term " $K$ is $n$-adjacent to the unknot in $V$ " to mean the following: There exists an embedding of $K$ in $V$ that contains $n$ generalized crossings such that changing any $0<m \leq n$ of them transforms $K$ into a knot that bounds an embedded disc in intV.

Recall that if $K$ is a non-trivial satellite with companion knot $\hat{C}$ and model knot $\hat{K}$ then:

i) $\hat{C}$ is non-trivial;

ii) $\hat{K}$ is geometrically essential in a standardly embedded solid torus $V_{1} \subset S^{3}$ but not isotopic to the core of $V_{1}$; and

iii) there is a homeomorphism $h: V_{1} \longrightarrow V:=h\left(V_{1}\right)$, such that $h(\hat{K})=K$ and $\hat{C}$ is the core of $V$.

The following theorem will be used to ensure that for every $n>0$ there is a hyperbolic knot that is $n$-adjacent to the unknot.

Theorem 2.2 ([10], Theorem 1.3) Let $K$ be a non-trivial satellite knot and let $V$ be any companion solid torus of $K$. If $K$ is $n$-adjacent to the unknot, for some $n>0$, then it is $n$-adjacent to the unknot in $V$. Furthermore, any model knot of $K$ is $n$-adjacent to the unknot in the standard solid torus $V_{1}$. 
The next result compiles the statements of Theorems 4.5, 5.2 and 5.6 of [2].

Theorem 2.3 2 2 Suppose that $K$ is a knot that is $n$-adjacent to the unknot for some $n>2$. Then, the following are true:

(a) All finite type invariants of orders less than $2 n-1$ vanish for $K$.

(b) We have $\Delta_{K}(t)=1$.

Given a knot $K$, a collection of crossing circles $K_{1}, \ldots, K_{n}$ and an $n$-tuple of integers $\mathbf{r}:=\left(r_{1} \ldots, r_{n}\right)$, let $K(\mathbf{r})$ denote the knot obtained from $K$ by performing a generalized crossing of order $\left|r_{i}\right|$ along $K_{i}$, for all $1 \leq i \leq n$.

Lemma 2.4 Suppose that $K$ is $n$-adjacent to the unknot, for some $n>1$, and that the crossing circles $K_{1}, \ldots, K_{n}$ constitute an $n$-trivializer for $K$. Then, for every $\mathbf{r}$ as above, $K(\mathbf{r})$ is also $n$-adjacent to the unknot.

Proof It follows immediately from Theorem 4.4 and Remark 5.5 of [2].

For a knot $K$ that is $n$-adjacent to the unknot and an $n$-trivializer $L_{n}:=$ $K_{1} \cup \ldots \cup K_{n}$ let $\eta\left(L_{n} \cup K\right)$ denote a tubular neighborhood of $L_{n} \cup K$. We close this section with two technical lemmas that we need in the next section.

Lemma 2.5 Suppose that $K$ is a non-trivial knot that is $n$-adjacent to the unknot, for some $n>0$. Then for every $n$-trivializer $L_{n}$, the 3 -manifold $M\left(K, L_{n}\right):=S^{3} \backslash \eta\left(L_{n} \cup K\right)$ is irreducible and $\partial$-incompressible.

Proof Let $D_{1}, \ldots, D_{n}$ be crossing discs bounded by $K_{1}, \ldots, K_{n}$, respectively. Assume, on the contrary, that $M\left(K, L_{n}\right)$ contains an essential 2-sphere $\Sigma$. Assume that $\Sigma$ has been isotoped so that the intersection $I:=\Sigma \cap\left(\cup_{i=1}^{n} D_{i}\right)$ is minimal. We must have $I \neq \emptyset$ since otherwise $\Sigma$ would bound a 3 -ball in $M\left(K, L_{n}\right)$. Let $c \in\left(\Sigma \cap D_{i}\right)$ denote a component of $I$ that is innermost on $\Sigma$; it bounds a disc $E \subset \Sigma$ whose interior is disjoint from $\cup_{i=1}^{n} D_{i}$. Since $\Sigma$ is separating in $M\left(K, L_{n}\right), E$ can't contain just one point of $K \cap D_{i}$ and, by the minimality assumption, $E$ can't be disjoint from $K$. Hence $E$ contains both points of $K \cap D_{i}$ and so $c=\partial E$ is parallel to $\partial D_{i}$ in $D_{i} \backslash K$. It follows that $K_{i}$ bounds an embedded disc in the complement of $K$. But then no twist along $K_{i}$ can unknot $K$, contrary to our assumption that $K_{i}$ is part of an $n$-trivializer of $K$. This contradiction finishes the irreducibility claim. To finish the proof of the lemma, suppose that $M\left(K, L_{n}\right)$ is $\partial$-compressible. Then, a component $T$ of $\partial M\left(K, L_{n}\right)$ admits a compressing disc $E^{\prime}$. Since $K$ is non-trivial, we must have $T=\partial \eta\left(K_{i}\right)$, for some $1 \leq i \leq n$. But then, $\partial E$ must be a longitude of $\eta\left(K_{i}\right)$. Thus $K_{i}$ bounds a disc in the complement of $K$; a contradiction. 
Lemma 2.6 Suppose that $K$ is a knot that is embedded in a standard solid torus $V_{1} \subset S^{3}$ so that it is geometrically essential in $V_{1}$. Suppose, moreover, that $K$ is $n$-adjacent to the unknot in $V_{1}$, for some $n>1$. Then, $K$ is not the unknot.

Proof Let $L_{n}$ be an $n$-trivializer that exhibits $K$ as $n$-adjacent to the unknot in $V_{1}$. Let $\mathcal{D}$ denote the disjoint union of a collection of crossing discs corresponding to the components of $L_{n}$. Suppose, on the contrary, that $K$ is the unknot. By Lemma 4.1 and the discussion in Remark 5.5 of [2] $K$ bounds an embedded disc $\Delta$ in the complement of $L_{n}$. Furthermore, $\Delta \cap \mathcal{D}$ is a collection $\mathcal{A}$ of $n$ disjoint properly embedded arcs in $\Delta$; one for each component of $\mathcal{D}$. Now making a crossing change on $K$ supported on a component $K_{i} \subset L_{n}$ is the same as twisting $\Delta$ along the arc in $\mathcal{A}$ corresponding to $K_{i}$. Since $n>1$, $\mathcal{A}$ has at least two components, say $\alpha_{1}, \alpha_{2}$, which cut $\Delta$ into three subdiscs. Since $K$ is geometrically essential in $V$, but can be unknotted by twisting along either of $\alpha_{1}, \alpha_{2}$, exactly two of these subdiscs must intersect $\partial V_{1}$ in a longitude. Recall that $\alpha_{1}, \alpha_{2}$ correspond to crossings that exhibit $K$ as 2-adjacent to the unknot in $V_{1}$. Thus $K$ can be also be uknotted by performing the aforementioned twists simultaneously on $\alpha_{1} \cup \alpha_{2}$. A straightforward checking will convince the reader that this is impossible.

\section{Knot adjacency and hyperbolic volume}

\subsection{Trivializers and hyperbolic Dehn filling}

In this section we will prove the main results of this note. As we will show Theorem 1.2 follows from the following theorem and known hyperbolic volume techniques.

Theorem 3.1 For every $n>2$, there exists a knot $\hat{K}$ that is $n$-adjacent to the unknot and it admits an $n$-trivializer $L_{n}$ such that the interior of $M_{n}:=M\left(\hat{K}, L_{n}\right)$ admits a complete hyperbolic structure with finite volume. Furthermore, we have $\operatorname{vol}\left(M_{n}\right)>n v_{3}$, where $v_{3}(\approx 1.01494)$ is the volume of a regular hyperbolic ideal tetrahedron.

The proof of Theorem 3.1 uses Thurston's uniformization theorem for Haken 3 -manifolds [18] in combination with a result of Adams [1]. Having Theorem 3.1] at hand, Theorem 1.2 follows from Thurston's hyperbolic Dehn filling theorem. 
The statements of these results of Thurston can also be found in [4. By abusing the terminology, we will say $M_{n}$ is hyperbolic to mean that the interior of $M_{n}$ admits a complete hyperbolic structure with finite volume.

Next let us show how Theorem 1.2 follows from Theorem [3.1. For the convenience of the reader we repeat the statement of Theorem 1.2

Theorem 3.2 For every $n \in \mathbf{N}$, there exists a hyperbolic knot $K$ such that:

(a) We have $\Delta_{K}(t)=1$.

(b) All the finite type invariants of orders $<2 n-1$ vanish for $K$.

(c) We have $\operatorname{vol}\left(S^{3} \backslash K\right)>n$.

(d) We have genus $(K)>\frac{n}{6}$.

Proof First assume that $n \leq 2$. Then, we have $2 n-1 \leq 3$ and thus to satisfy part(c) we need a knot that has trivial finite type invariants of orders $\leq 2$. It is known that the only finite type invariant of order $\leq 2$ is essentially the second derivative of the Alexander polynomial at $t=1$. Thus, a knot with trivial Alexander polynomial has trivial finite type invariants of order $\leq 2$. There are hyperbolic knots with trivial Alexander polynomial; the first such knots occur at thirteen crossings and all have volume $>2$. Thus for $n \leq 2$ there are hyperbolic knots satisfying parts (a)-(c). But note that (d) is trivially satisfied. Suppose now that $n>2$. By Theorem 3.1 there exists a non-trivial knot $\hat{K}$, that is $n$-adjacent to the unknot, and an $n$-trivializer, say $L_{n}$, so that $M_{n}$ is hyperbolic and $\operatorname{vol}\left(M_{n}\right)>n v_{3}$. Let $K_{1}, \ldots, K_{n}$ denote the components of $L_{n}$. Given an $n$-tuple of integers $\mathbf{r}:=\left(r_{1}, \ldots, r_{n}\right)$, let $M_{n}(\mathbf{r})$ denote the 3 -manifold obtained from $M_{n}$ as follows: For $1 \leq i \leq n$, perform Dehn filling with slope $\frac{1}{r_{i}}$-surgery along $\partial \eta\left(K_{i}\right)$. Let $\hat{K}(\mathbf{r})$ denote the image of $\hat{K}$ in $M_{n}(\mathbf{r})$. Clearly, $M_{n}(\mathbf{r}):=S^{3} \backslash \eta(\hat{K}(\mathbf{r}))$ and $\hat{K}(\mathbf{r})$ is obtained from $\hat{K}$ by generalized crossing changes. By Lemma 2.4 $\hat{K}(\mathbf{r})$ is $n$-adjacent to the unknot, for every $n$-tuple $\mathbf{r}$. On the other hand, by Thurston's hyperbolic Dehn filling theorem, if $r_{i}>>0$ then $M_{n}(\mathbf{r})$ admits a complete hyperbolic structure of finite volume; thus $\hat{K}(\mathbf{r})$ is a hyperbolic knot. By the proof of Thurston's theorem, the hyperbolic metric on $M_{n}(\mathbf{r})$ can be chosen so that it is arbitrarily close to the metric of $M_{n}$, provided that the numbers $r_{i}$ are all sufficiently large. Thus by choosing the $r_{i}$ 's large we may ensure that the volume of $M_{n}(\mathbf{r})$ is arbitrarily close to that of $M_{n}$. Since $\operatorname{vol}\left(M_{n}\right)>n v_{3}$, we can choose the $r_{i}$ 's so that we have $\operatorname{vol}\left(M_{n}(\mathbf{r})\right)>n$. But since $\operatorname{vol}\left(M_{n}(\mathbf{r})\right)=\operatorname{vol}\left(S^{3} \backslash \hat{K}(\mathbf{r})\right)$, setting $K:=\hat{K}(\mathbf{r})$ we are done. This finishes the proof of parts (a)-(c) of the theorem. For part (d), recall that by the main result of [9] (or by Theorem 1.3 of [11]), we have 6 genus $(K)-3 \geq n$. Thus, $\operatorname{genus}(K) \geq \frac{n+3}{6}>\frac{n}{6}$ as desired. 


\subsection{Ensuring hyperbolicity}

The rest of the section is devoted to the proof of Theorem 3.1. Our purpose is to show that given $n>2$, we can find a non-trivial knot $\hat{K}$, that is exhibited $n$ adjacent to the unknot by an $n$-trivializer $L_{n}$ such that the 3 -manifold $M_{n}$ := $S^{3} \backslash \eta\left(\hat{K} \cup L_{n}\right)$ has the following properties:

(i) $M_{n}$ is irreducible.

(ii) $M_{n}$ is $\partial$-incompressible.

(iii) $M_{n}$ is atoroidal; every incompressible embedded torus is parallel to a component of $\partial M_{n}$.

(iv) $M_{n}$ is anannular; every incompressible properly embedded annulus can be isotoped on $\partial M_{n}$.

Having properties (i)-(iv) at hand, we can apply Thurston's uniformization theorem for Haken 3-manifolds to conclude that $M_{n}$ is hyperbolic. Now $\partial M_{n}$ is a collection of tori. Associated with each component of $\partial M_{n}$, there is a cusp in $\operatorname{int} M_{n}$ homeomorphic to $T^{2} \times[1, \infty)$. A result of Adams [1] states that a complete hyperbolic 3-manifold with $N$ cusps must have volume at least $N$. $v_{3}$. Since $\partial M_{n}$ has $n+1$ components, $\operatorname{int} M_{n}$ has $n+1$ cusps. Thus $\operatorname{vol}\left(M_{n}\right) \geq(n+1) v_{3}>n v_{3}$ and the desired conclusion follows.

By [2], for every $n \in \mathbf{N}$, there exist many non-trivial knots that are $n$-adjacent to the unknot. Fix $n>2$. Let $\bar{K}$ be any non-trivial knot that is $n$-adjacent to the unknot and let $L_{n}$ be any $n$-trivializer corresponding to $\bar{K}$. By lemma 2.5. properties (i)-(ii) hold for $M_{n}:=S^{3} \backslash \eta\left(\bar{K} \cup L_{n}\right)$. Next we set off to show that we can choose $\bar{K}$ and $L_{n}$ so that $M_{n}$ satisfies properties (iii)-(iv) as well. We need the following lemma that will be used to control essential tori in the complement of $n$-trivializer's.

Lemma 3.3 Let $L_{n}$ be an $n$-trivializer that shows a non-trivial knot $\bar{K}$ to be $n$-adjacent to the unknot. Suppose that $M_{n}:=S^{3} \backslash \eta\left(L_{n} \cup \bar{K}\right)$ contains an essential torus $T$ and let $V$ denote the solid torus in $S^{3}$ bounded by $T$. Then, $L_{n}$ can be isotoped in int $V$ by an isotopy in the complement of $\bar{K}$. Furthermore, $T$ remains essential in the complement of $\bar{K}$.

Proof First we show that $L_{n}$ can be isotoped in $\operatorname{int} V$, by an isotopy in the complement of $\bar{K}$. Suppose that a component $K_{1} \subset L_{n}$ lies outside $V$ and let $D_{1}$ be a crossing disc bounded by $K_{1}$. We can isotope $D_{1}$ so that every component of $D_{1} \cap T$ is essential in $D_{1} \backslash \bar{K}$. Thus, each component of $D_{1} \cap T$ is 
either parallel to $\partial D_{1}$ on $D_{1}$, or it bounds a subdisc of $D_{1}$ that is intersected exactly once by $\bar{K}$. In the first case, $K_{1}$ can be isotoped to lie in $\operatorname{int} V$. In the later case, each component of $D_{1} \cap T$ bounds a subdisc $E \subset D_{1}$ that is intersected exactly once by $\bar{K}$. Since $T$ is essential in the complement of $\bar{K}, E$ must be a meridian disc of $V$. Thus $\bar{K}$ has wrapping number one in the follow-swallow torus. It follows that $\bar{K}$ is composite and that the crossing change corresponding to $L_{1}$ occurs within a factor of $\bar{K}$. But then such a crossing change cannot unknot $\bar{K}$ contradicting the fact that $L_{1}$ is part of an $n$-trivializer. Thus $L_{n}$ can be isotoped to lie in $\operatorname{int} V$ as desired. Now it is easy to see that $L_{n}$ exhibits $\bar{K}$ as $n$-adjacent to the unknot in $\operatorname{int} V$.

Next we show that $T$ remains essential in the complement of $\bar{K}$.

Since the linking number of $\bar{K}$ and each component of $L_{n}$ is zero, $\bar{K}$ bounds a Seifert surface in the complement of $L_{n}$. Let $S$ be a Seifert surface of $\bar{K}$ that is of minimum genus (and thus of maximum Euler characteristic) among all such Seifert surfaces of $\bar{K}$. Since $T, S$ are incompressible in $M_{n}$ we can isotope them so that $S \cap T$ is a collection of parallel essential curves on $T$. Since twisting along any component of $L_{n}$ unknots $\bar{K}$, the winding number $\bar{K}$ in $V$ is zero. We conclude that $S \cap T$ is homologically trivial in $T$. Thus we may replace the components of $S \backslash T$ that lie outside $V$ by annuli on $T$, and then isotope these annuli away from $T$ in $V$, to obtained a Seifert surface $S^{\prime}$ for $\bar{K}$. Now $S^{\prime}$ lies in $M_{n} \cap V$. Since no component of $X:=S \cap \overline{S^{3} \backslash V}$ can be a disc, the Euler characteristic $\chi(X)$ is not positive. Thus $\chi\left(S^{\prime}\right) \geq \chi(S)$. Since $S$ was chosen to be of maximum Euler characteristic for $K$ in the complement of $L_{n}$, we have $\chi\left(S^{\prime}\right) \leq \chi(S)$. It follows that $\chi\left(S^{\prime}\right)=\chi(S)$ and thus $\operatorname{genus}\left(S^{\prime}\right)=\operatorname{genus}(S)$. Hence $S^{\prime}$ is also a minimum genus Seifert surface for $\bar{K}$ in $M_{n}$. Let $\mathcal{D}$ be a collection of crossing discs; one for each component of $L_{n}$. For $D \in \mathcal{D}$, the components of $S^{\prime} \cap D$ are closed curves that are parallel to $\partial D$ on $D$ and an arc properly embedded on $S^{\prime}$. After an isotopy of $S^{\prime}$ we can arrange so that $S^{\prime} \cap D$ is only an arc $\alpha$ properly embedded in $S^{\prime}$. Thus, $S^{\prime} \cap \mathcal{D}$ is a collection, say $\mathcal{A}$ of arcs properly embedded on $S^{\prime}$. By Theorem 4.1 of $[9], S^{\prime}$ remains of minimum genus in the complement of $\bar{K}$. That is, genus $(\bar{K})=\operatorname{genus}\left(S^{\prime}\right)$. Thus $S^{\prime}$ is incompressible in the complement of $\bar{K}$. By assumption, twisting along all the components of $L_{n}$ turns $\bar{K}$ into the unknot in $V$. This unknot can be isotoped to lie in a 3 -ball $B \subset \operatorname{int} V$. Assume that $\bar{K}$ is inessential in $V$; then $\bar{K}$ can also be isotoped to lie in $B$. Since $S^{\prime}$ is incompressible, $S^{\prime}$ can also be isotoped to lie in $B$. But then, $\mathcal{A}$ and $\bar{K}$ will lie in $B$. Now since $L_{n}$ can be isotoped so that it lies in a small neighborhood of $\mathcal{A}$ in a collar of $S^{\prime}$, it follows that $\bar{K} \cup L_{n}$ can be isotoped in $B$. But this contradicts the assumption that $T$ is essential in $M_{n}$. 
Next we turn our attention to essential annuli in $M_{n}$. It is known that an atoroidal link complement that contains essential annuli admits a Seifert fibration over a sphere with at most three punctures. In particular such a link can have at most 3 -components. Hence, the restriction $n>2$ in our case implies that if $M_{n}$ is atoroidal then it is anannular. For the convenience of the reader we give a direct argument that $M_{n}$ is anannular in the next lemma.

Lemma 3.4 Let $L_{n}$ be an $n$-trivializer of a non-trivial knot $\bar{K}$. If $n>2$ and $M_{n}$ is atoroidal, then $M_{n}$ is anannular.

Proof Since $\bar{K}$ is non-trivial, by Lemma 2.5. $M_{n}$ is irreducible. We will show that every incompressible, properly embedded annulus $(A, \partial A) \hookrightarrow\left(M_{n}, \partial M_{n}\right)$ can be isotoped to lie on $\partial M_{n}$.

First, suppose that $A$ runs between two components of $\partial M_{n}$, say $T_{1}, T_{2}$. Let $N$ denote a neighborhood of $A \cup T_{1} \cup T_{2}$. Now $\partial N$ has three tori components. Two of them are parallel to $T_{1}, T_{2}$, respectively. Let $T$ denote the third one. Since $n>2, \partial M_{n}$ has at least four components. Thus $T$ separates pairs of components of $\partial M_{n}$ and it can't be boundary parallel. Hence, since $M_{n}$ is atoroidal, $T$ must be compressible. A compressing disc cuts $T$ into a 2sphere that separates pairs of components of $\partial M_{n}$ and thus it is essential; a contradiction. Therefore, $\partial A$ must lie on one component, say $T_{1}$, of $\partial M_{n}$. Let $N$ denote a neighborhood on $A \cup T_{1}$. Now $\partial N$ contains a torus $T$ that separates $T_{1}$ from the rest of the components of $\partial M_{n}$. Suppose, for a moment, that $T$ compresses. Then a compressing disc cuts $T$ into a 2 -sphere that separates $T_{1}$ from the rest of the components of $\partial M_{n}$. But such a sphere would be essential contradicting the irreducibility of $M_{n}$. Therefore we conclude that $T$ must be parallel to a component of $\partial M_{n}$. But since $T$ separates $T_{1}$ from at least three components of $\partial M_{n}$, it can only be parallel to $T_{1}$. Hence $A$ is contained in a collar $T_{1} \times I$ and it can be isotoped on $T_{1}$. This proves that $A$ is inessential which finishes the proof of the lemma.

\subsection{Completing the proofs}

We are now ready to give the proofs of the main results. We begin with the proof of Theorem 3.1

Theorem 3.5 For every $n>2$, there exists a knot $\hat{K}$ that is $n$-adjacent to the unknot and it admits an $n$-trivializer $L_{n}$ such that the interior of $M_{n}:=M\left(\hat{K}, L_{n}\right)$ admits a complete hyperbolic structure with finite volume. 
Furthermore, we have $\operatorname{vol}\left(M_{n}\right)>n v_{3}$, where $v_{3}(\approx 1.01494)$ is the volume of a regular hyperbolic ideal tetrahedron.

Proof To complete the proof we need the following claim:

Claim For every $n>2$, there is a non-trivial knot $\hat{K}$ that is not a satellite (i.e. its complement is atoroidal) and it is $n$-adjacent to the unknot.

Proof of Claim By 2] there is a non-trivial knot $\bar{K}$ that is $n$-adjacent to the unknot. Suppose $\bar{K}$ is a satellite knot. By applying Theorem 2.2 inductively we can find a pattern knot $\hat{K}$ of $\bar{K}$ such that i) $\hat{K}$ is not a non-trivial satellite; and ii) $\hat{K}$ is $n$-adjacent to the unknot in a standard solid torus $V_{1}$. By Lemma [2.6. $\hat{K}$ is not the unknot and the claim is proved.

To continue with the proof of the theorem, fix $n>2$ and let $\bar{K}$ be a knot as in the claim above. Also let $L_{n}$ be an $n$-trivializer for $\hat{K}$. By Lemma 2.5. $M_{n}:=S^{3} \backslash \eta\left(L_{n} \cup \bar{K}\right)$ is irreducible and $\partial$-incompressible. We claim that $M_{n}$ is atoroidal. For, suppose that $M_{n}$ contains an essential torus. By Lemma 3.3 $T$ must remain essential in the complement of $\hat{K}$. But this is impossible since $\hat{K}$ was chosen to be non-satellite. Hence $M_{n}$ is atoroidal and by Lemma 3.4 anannular. Now Thurston's uniformization theorem applies to conclude that $M_{n}$ is hyperbolic. Since $\partial M_{n}$ has $n+1$ components, the interior of $M_{n}$ has $n+1$ cusps. By [1], $\operatorname{vol}\left(M_{n}\right) \geq(n+1) v_{3}$ and thus $\operatorname{vol}\left(M_{n}\right)>n v_{3}$ as desired.

As we proved earlier, Theorem 1.2 follows from Theorem 3.1. Note that Corollary 1.1 follows immediately from Theorem 1.2. We will finish the section with the proof of Corollary 1.3

Corollary 3.6 For every $n, m \in \mathbf{N}$, there exists a hyperbolic knot $K$ with trivial finite type invariants of orders $\leq m$ and such that $\operatorname{vol}\left(S^{3} \backslash K\right)>n$.

Proof Fix $m \in \mathbf{N}$. By Theorem [1.2, there is a hyperbolic knot $K$ with $\operatorname{vol}\left(S^{3} \backslash K\right)>m$ and with trivial finite type invariants of orders $\leq m$. Now this $K$ works for all $n$ with $n<m$. To obtain a knot corresponding to $m, n$ for some $n>m$ just apply Theorem 1.2 for this $n$.

\section{Concluding discussion}

\subsection{Mahler measure of Alexander polynomials and volume}

For a hyperbolic link $L$ with an unknotted component $K$ let $L(q)$ denote the link obtained from $L \backslash K$ by a twist of order $q$ along $K$. Thurston's hyperbolic 
Dehn filling theorem implies that as $q \longrightarrow \infty$, the volume of the complement of $L(q)$ converges to the volume of the complement of $L$. In [15, D. Silver and S. Williams showed that the Mahler measure of the Alexander polynomial of $L(q)$ exhibits an analogous behavior; it converges to the Mahler measure of the Alexander polynomial of $L$, as $q \longrightarrow \infty$. This analogy prompted the question of whether the Mahler measure of the Alexander polynomial of a hyperbolic knot is related to the volume of its complement [15. In that spirit, Corollary 1.1 provides examples of non-alternating, hyperbolic knots with arbitrarily large volume whose Alexander polynomial has trivial Mahler measure. In [16, Silver, Stoimenow and Williams construct examples of alternating knots with these properties. However they also show that, for alternating knots, large Mahler measure of the Alexander polynomial implies large volume of the complement.

\subsection{The Jones polynomial and volume}

Corollary 1.1 shows that the Alexander polynomial of generic (non-alternating) hyperbolic knots is far from controlling the hyperbolic volume. The determinant of a knot can also be evaluated from its Jones polynomial. More specifically, if $J_{K}(t):=\sum_{i=0}^{r} a_{i} t^{i}$ denotes the Jones polynomial of an alternating knot $K$, then $\operatorname{det}(K):=\left|J_{K}(-1)\right|=\sum_{i=0}^{r}\left|a_{i}\right|$. Thus, the inequalities in (4) can also be expressed in terms of the Jones polynomial of $K$. In particular, the right hand side inequality becomes $\operatorname{vol}\left(S^{3} \backslash K\right) \leq C\left(\sum_{i=0}^{r}\left|a_{i}\right|\right)$. A recent result O. Dasbach and X.-S. Lin [7, which preceded and inspired the results in this note, gives a striking relation between the Jones polynomial and the volume of alternating hyperbolic knots. More specifically, the Volume-ish theorem of [7] states that the volume of the complement of a hyperbolic alternating knot is bounded above and below by linear functions of absolute values of certain coefficients of the Jones polynomial of the knot. Furthermore, the authors record experimental data on the correlation between the coefficients of the Jones polynomial and the volume of knots up to 14 crossings. For generic knots, this data suggests a better correlation than the one between the coefficients of Alexander polynomial and volume. In the view of the experimental evidence of [7], and the examples constructed in this note, it becomes interesting to study the Jones polynomial of knots that are $n$-adjacent to the unknot, for $n \gg 0$.

\section{References}

[1] C. C. Adams, Volumes of $N$-cusped hyperbolic 3 -manifolds, J. London Math. Soc. (2) 38 (1988), no. 3, 555-565. MathReview 
[2] N. Askitas, E. Kalfagianni, On knot adjacency, Topology and its Applications, Vol. 126 (2002), 63-81. MathReview

[3] D. Bar-Natan, S. Garoufalidis, On the Melvin-Morton-Rozansky conjecture, Invent. Math. 125 (1996), no. 1, 103-133. MathReview

[4] F. Bonahon, Geometric structures on 3-manifolds, Handbook of geometric topology, 93-164, North-Holland, Amsterdam, 2002. MathReview

[5] G. Burde, H. Zieschang, Knots. de Gruyter Studies in Mathematics, 5. Walter de Gruyter \& Co., Berlin, 1985. MathReview

[6] P. Callahan, A. Reid, Hyperbolic structures on knot complements. Knot theory and its applications. Chaos Solitons Fractals 9 (1998), no. 4-5, 705-738. MathReview

[7] O. Dasbach, X.-S. Lin, A volume-ish theorem for the Jones polynomial of alternating knots, arXiv:math.GT/0403448

[8] D. Gabai, Foliations and the topology of 3-manifolds III, J. Diff. Geom. vol 26 (1987), 479-536. MathReview

[9] H. Howards, J. Luecke, Strongly $n$-trivial knots, Bull. London Math. Soc. 34 (2002), no. 4, 431-437. MathReview

[10] E. Kalfagianni, X.-S. Lin, Knot adjacency and satellites, Topology and its Applications, Vol. 138 (2004), 207-217. MathReview

[11] E. Kalfagianni, X.-S. Lin, Knot adjacency, genus and essential tori, (with an appendix by D. McCullough), arXiv:math.GT/0403024

[12] R. M. Kashaev, The hyperbolic volume of knots from the quantum dilogarithm, Lett. Math. Phys. 39 (1997), no. 3, 269-275. MathReview

[13] W. Menasco, Closed incompressible surfaces in alternating knot and link complements, Topology 23 (1984), no. 1, 37-44. MathReview

[14] H. Murakami, J. Murakami, The colored Jones polynomials and the simplicial volume of a knot, Acta Math. 186 (2001), no. 1, 85-104. MathReview

[15] D. Silver, S. Williams, Mahler measure of Alexander polynomials, J. London Math. Soc. (2) 69 (2004), no. 3, 767-782. MathReview

[16] D. Silver, A. Stoimenow, S. Williams, Euclidean Mahler measure and twisted links, in preparation.

[17] A. Stoimenow, Graphs, determinants of knots and hyperbolic volume, preprint, 2003.

[18] W. Thurston, The Geometry and Topology of 3-manifolds. Available at http://www.msri.org/publications/books/gt3m

Department of Mathematics, Michigan State University, E. Lansing, MI 48824, USA

or

School of Mathematics, Institute for Advanced Study, Princeton, NJ 08540, USA

Email: kalfagia@math.msu.edu or kalfagia@math.ias.edu

URL: http://www.math.msu.edu/ kalfagia

Received: 22 September 2004 